\title{
Cornell Scale for Depression in Dementia
}

National Cancer Institute

\section{Source}

National Cancer Institute. Cornell Scale for Depression in Dementia. NCI Thesaurus.

Code C155929.

A 19-item clinician-administered instrument, developed by Alexopoulos et al. in 1986, that utilizes a three-point rating scale to assess signs and symptoms of major depression in patients with dementia, based on interviews with both the patient and a caregiver. 